https://doi.org/10.15407/ujpe66.7.619

L.I. MALYSHEVA

Bogolyubov Institute for Theoretical Physics, Nat. Acad. of Sci. of Ukraine

(14b, Metrologichna Str., Kyiv 03680, Ukraine; e-mail: malysh@bitp.kiev.ua)

\title{
ANALYTIC ANALYSIS OF ELECTRONIC AND TRANSPORT PROPERTIES OF FINITE POLYENES
}

\begin{abstract}
Various important characteristics of finite polyene chains are found on the basis of approximate solutions of the characteristic equations. The obtained approximate and limit expressions for the wave functions, energy gap, etc. can be used for the analysis of the electronic and transport properties of polyenes, which gives a deeper understanding of the fundamental properties of finite alternating polyene chains. We also demonstrate the high efficiency of the proposed approximations as a zero-order estimate for the numerical solution of the characteristic equation.

Keywords: polyene, Green functions, energy gap, transmission coefficient.
\end{abstract}

\section{Introduction}

Theoretical investigations of polyacetylenes (as potentially conducting polymers) and finite oligopolyenes of known length were carried out in numerous works; see, e.g., the review [1] and the references therein. The applications of polyenes in the field of nanoelectronics is explained by their controllable chemical and electrochemical properties. A simple model that includes the first and second excited singlet states of linear polyenes and thus explains their experimentally measured electronic properties, e.g., the nonlinear optical response, was proposed 30 years ago [2]. With an aim to construct the analytic theory of nonlinear response within the framework of the model proposed in [2], exact equations for the one-electron molecular orbital coefficients for an alternating polyene chain were proposed and solved in [3]. Later, explicit expressions for the matrix elements of the Green function for bounded polyenes were deduced in $[4,5]$. In addition to analytic investigations, numerous studies were focused on the numerical methods: the Green function method in combination with DFT calculations were used to compute the electron and transport characteristics of cyclic and linear polyenes [6]; high-precision DTF calculations of the structure and harmonic frequencies of polyenes were performed in [7]. In the present paper, we propose an analytic approach to the analysis of the electronic and transport properties and other fundamental problems arising in the theory of bounded polyenes.

(C) L.I. MALYSHEVA, 2021

ISSN 2071-0194. Ukr. J. Phys. 2021. Vol. 66, No. 7
The electronic structure of a bounded polyene chain is determined by the solutions of the characteristic equation. The model-exact equation for the eigenvalues of the system depicted in Fig. 1 appears in the Lennard-Jones theory of polyenes [8]. Recently, the solutions of the characteristic equation were analyzed for bounded graphene sheets and linear polyenes, and several approximate solutions were proposed [9]. The obtained analytic approximations turn out to be very accurate and are used in what follows to find the expressions for eigenvalues and eigenstates of finite polyene chains. Thus, in order to determine the eigenstates and Green functions of the bounded polyene chain presented in Fig. 1 and, hence, to clarify its various electronic and transport properties, we use approximate solutions of the transcendental characteristic equation to get reliable approximations to various important characteristics of polyenes: energy gaps, wave functions, and transmission probability.

\section{Eigenvalues, Eigenstates, and Green Functions of Finite Polyene Chains}

The simplest and most successfully parametrized oneelectron Hamiltonian used for the description of the $\pi$-electron electronic structure of linear polyene, i.e., a bounded chain of $N \mathrm{CH}$ groups; see Fig. 1, is the Hückel Hamiltonian. The Su-Schrieffer-Heeger model of polyene chains [10] uses two parameters: the electron resonance transfer energy between the nearest-neighbor $\mathrm{CH}$ groups in the undimerized polyene $t_{0}\left(t_{0}<0\right)$ and its variation caused by the 


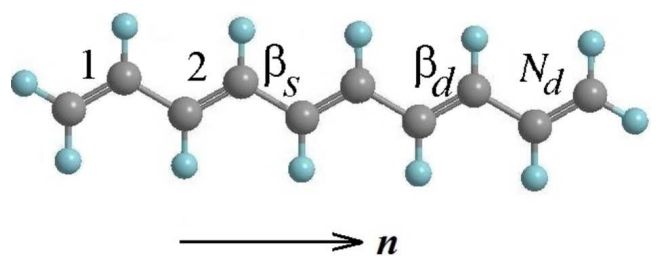

Fig. 1. Bounded polyene chain with $N_{d}=N / 2$ double bonds; $\beta_{s}$ and $\beta_{d}$ specify the energy of electron transfer between the carbon atoms connected by single and double bonds, respectively; $n$ numbers the carbon atoms.

dimerization $\pm k u$ expressed in terms of the electronphonon coupling constant $k$ and the $\mathrm{C}-\mathrm{C}$-bond alternation $u$ along the axis of the molecule. Then the hopping integrals $\beta_{s}$ and $\beta_{d}$ specifying the energy of electron transfer between the nearest neighbors connected by single and double bonds, respectively, can be represented via the following two new parameters:

$\beta_{s}=\beta e^{-\eta}, \quad \beta_{d}=\beta e^{\eta}$,

related to the parameters $u, k$, and $t_{0}$ by the following equations:

$t_{0}=\beta \cosh \eta, \quad k u=\beta \sinh \eta$.

A similar approach based on the use of the hopping integral weighted with an exponential function has been recently applied to the analysis of the transport properties of $\pi$-conjugated molecules [11].

Thus, a chain of $\mathrm{CH}$ groups with alternating length of $\mathrm{C}-\mathrm{C}$ bonds is described by the electronic part of the $\mathrm{Su}$-Schrieffer-Heeger Hamiltonian [10]

$\hat{H}^{\mathrm{PE}}=-\beta_{d} \sum_{m=1}^{N_{d}}\left(\left|m_{l}\right\rangle\left\langle m_{r}\right|\right)-$

$-\beta_{s} \sum_{m=1}^{N_{d}}\left(\left|(m+1)_{l}\right\rangle\left\langle m_{r}|+|(m-1)_{r}\right\rangle\left\langle m_{l}\right|\right)$.

In Eq. (1), the index $m$ runs over the double bonds whose number is equal to a half of the number of all carbon atoms in the chain, i.e., $N_{d}=N / 2$; the ket vector $\left|m_{l(r)}\right\rangle$ has the meaning of a $2 p_{z}$ atomic orbital of the carbon atom, the label $l(r)$ refers to the left (right) atom connected by the $m$ th double bond. $\left|0_{r}\right\rangle=\left|(N+1)_{l}\right\rangle=0 ;-\beta_{s}$ and $-\beta_{d}$ are the hopping integrals between the nearest neighbors connected by single and double bonds, respectively.
The solution of the Schrödinger equation with Hamiltonian (1) gives the $\pi$-electron spectrum formed by valence (v) and conduction (c) bands

$$
E_{\nu}^{\mathrm{v}(\mathrm{c})}=-(+) \sqrt{2\left(\cosh 2 \eta+\cos x_{\nu}\right)}, \quad \nu=1, \ldots, N_{d},
$$

in $\beta$ units. For open-end boundary conditions, i.e., for the $(\mathrm{C}=\mathrm{C})_{N_{d}}$ chain with $\mathrm{CH}_{2}$ terminal groups, the values $x_{\nu}$ are the solutions of the Lennard-Jones equation [8]

$D \equiv e^{-2 \eta} \sin \left(N_{d} x\right)+\sin \left[\left(N_{d}+1\right) x\right]=0$

within the interval $0 \leq x \leq \pi$. The approximate solutions of this equation are presented in the next section. These approximations can be used as zero-order values for the numerical solution of Eq. (3).

In the model of polyene $\pi$-electron spectrum described above, the appearance of the band gap is entirely due to the bond-length alternation (manifestation of the Peierls instability), which is described by the alternation parameter $\eta$. Note that, for $\eta=0$, we have $\hat{H}^{\mathrm{PE}} \rightarrow \hat{H}^{(\mathrm{C})_{N}}$. In this case, Eq. (3) gives $\left.x_{\nu}\right|_{\eta=0}=2 \pi \nu /\left(2 N_{d}+1\right)$.

The eigenstates of the Hamiltonian $\hat{H}^{\mathrm{PE}}$

$\Psi_{\mathrm{v}(\mathrm{c}), \nu}^{\mathrm{PE}}=\sum_{m=1}^{N_{d}}\left(\psi_{2 m-1, \nu}^{\mathrm{v}(\mathrm{c})}|2 m-1\rangle+\psi_{2 m, \nu}^{\mathrm{v}(\mathrm{c})}|2 m\rangle\right)$,

were found in [3] and have the form:

$\psi_{2 m-1, \nu}^{\mathrm{v}(\mathrm{c})}= \pm(-1)^{\nu+1} B\left(x_{\nu}\right) \sin \left[\left(N_{d}+1-m\right) x_{\nu}\right]$,

$\psi_{2 m, \nu}^{\mathrm{v}(\mathrm{c})}=B\left(x_{\nu}\right) \sin \left(m x_{\nu}\right)$,

where the normalization constant $B$ is

$B^{2}\left(x_{\nu}\right)=\frac{2 \sin x_{\nu}}{\left(2 N_{d}+1\right) \sin x_{\nu}-\sin \left[\left(2 N_{d}+1\right) x_{\nu}\right]}$.

The matrix elements of the Green function needed to find the transmission spectra are (in $\beta$ units) [5]

$G_{1,1}=G_{N, N}=\frac{E \sin \left(N_{d} x\right)}{D}, \quad G_{1, N}=-\frac{e^{\eta} \sin x}{D}$,

where $D$ is defined in Eq. (3) and

$2 \cos x+2 \cosh 2 \eta=E^{2}$.

In the next section, we obtain approximate expressions for the eigenvalues and eigenstates of finite polyene chains by using the approximate solutions of Eq. (3) recently found in [9].

ISSN 2071-0194. Ukr. J. Phys. 2021. Vol. 66, No. 7 


\section{Approximations and Limit Expressions for Finite Polyene Chains}

Using the results from [9], we find the following approximation for the eigenenergies of bounded polyene chain:

$x_{\nu} \approx \frac{\pi \nu}{N_{d}+1-\frac{1}{1+e^{2 \eta}}}$.

Despite the fact that this relation was obtained for $\nu \ll N_{d}$, it gives fairly accurate results for almost all $\nu$ except $\nu=N_{d}-\mu, \mu \ll N_{d}$. Thus, Eq. (10) can be successively applied as the zero-order approximation of solutions of Eq. (3) in the course of construction of the numerical solution of the characteristic equation.

In addition, for the most interesting case $\eta \ll 1$, Eq. (10) can be simplified to

$x_{\nu} \approx \frac{2 \pi \nu}{2 N_{d}+1+\eta}$

up to the order $O\left(\eta^{2}\right)$. This relation is used in what follows to find important characteristics of finite polyenes, such as the HOMO-LUMO gap $\Delta_{\mathrm{HL}}$ and wave functions. In order to obtain more accurate values of $x_{\nu}$ for $\nu$ close to $N_{d}$, we use a more sophisticated approximation found in [9]. Rewritten for the case of a polyene chain with $\eta \ll 1$, it has the form

$x_{\nu} \approx \frac{\pi(2 \nu-\eta)}{2 N_{d}+1+\eta}-\frac{\eta}{\pi} \frac{2-\pi^{2}\left(\eta^{2}+A\right)}{\eta+N_{d}\left(2 \eta^{2}+A\right)}$,

where

$A \equiv \frac{2 \mu+1+\eta(2 \mu+3)}{2 N_{d}+1}$.

In what follows, we use approximations (10)-(12) to find important characteristics of a polyene chain formed by $N$ carbon atoms, such as the HOMOLUMO gap $\Delta_{\mathrm{HL}}, \mathrm{HOMO}$ and $\mathrm{HOMO}-1$ gap $\left(\Delta_{\mathrm{HL}}^{++}\right)$, and wave functions.

\subsection{HOMO-LUMO gap}

By using relation (11), we find a simple approximation for $\Delta_{\mathrm{HL}}$, which works well for $N_{d} \gtrsim 3$ :

$\Delta_{\mathrm{HL}}=E_{N_{d}}^{\mathrm{c}}-E_{N_{d}}^{\mathrm{v}} \approx 2 \sqrt{\frac{\pi^{2}(1+2 \eta)}{\left(2 N_{d}+1\right)^{2}}+4 \eta^{2}}$

(for $N_{d}=1,2$, the exact relations for $\Delta_{\mathrm{HL}}$ can be easily found analytically; see the Appendix). In the limit

ISSN 2071-0194. Ukr. J. Phys. 2021. Vol. 66, No. 7

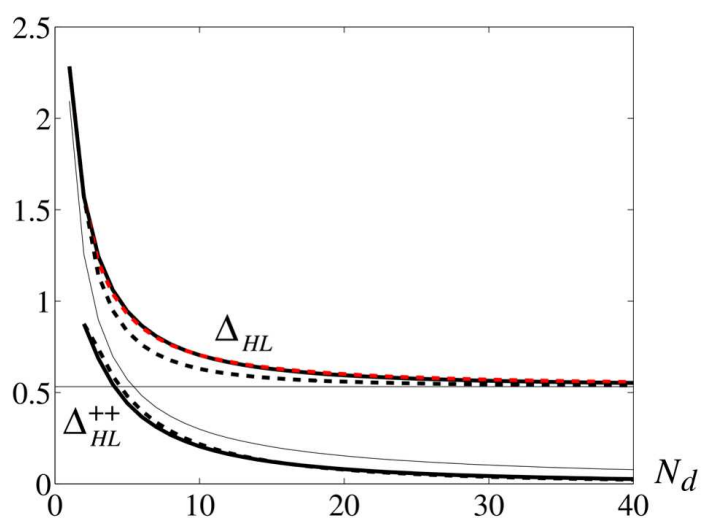

Fig. 2. The heavy lines: exact dependences of the HOMOLUMO gap $\Delta_{\mathrm{HL}}$ and HOMO and HOMO-1 gap $\left(\Delta_{\mathrm{HL}}^{++}\right)$(in the units of $\beta$ ) for a bounded polyene on $N_{d} ; \eta=0.133$ [3]. The black dashed lines: approximation (14) and relation (19) with $x_{\nu}$ found with the help of Eq. (11). The red dashed line: $\Delta_{\mathrm{HL}}$ with $x_{N_{d}}$ found with the help of Eqs. (12), (13). The thin lines: the limit expressions (15) and (17)

$N_{d} \rightarrow \infty$, Eq. (14) gives the following well-known result:

$\lim _{N_{d} \rightarrow \infty} \Delta_{\mathrm{HL}}=4 \eta$

In the opposite case of the smallest $N_{d}=1$ for which the exact value of $\Delta_{\mathrm{HL}}$ can be easily found and equals $2 e^{\eta}$, approximation (14) is also reasonable:

$\Delta_{\mathrm{HL}} \approx 2 \frac{\pi}{3} \sqrt{1+2 \eta}$

Thus, for small $N_{d}$, when the first term under the square-root sign in Eq. (14) is predominant:

$\Delta_{\mathrm{HL}} \approx \frac{2 \pi \sqrt{1+2 \eta}}{2 N_{d}+1}$

the values of the HOMO-LUMO gap are inversely proportional to $N_{d}$ and weakly depend on $\eta$. As $N_{d}$ increases, the second term $4 \eta^{2}$ becomes larger than the first term, and, for

$N_{d} \gg \frac{\pi}{4 \eta}$

the HOMO-LUMO gap is well approximated by Eq. (15). These trends are well visible in Fig. 2, where approximations (14), (15), and (17) are compared with the exact dependences for $\Delta_{\mathrm{HL}}\left(N_{d}\right)$.

It should be noted that the smaller $\nu$, the greater the accuracy of approximation (10). Thus, 

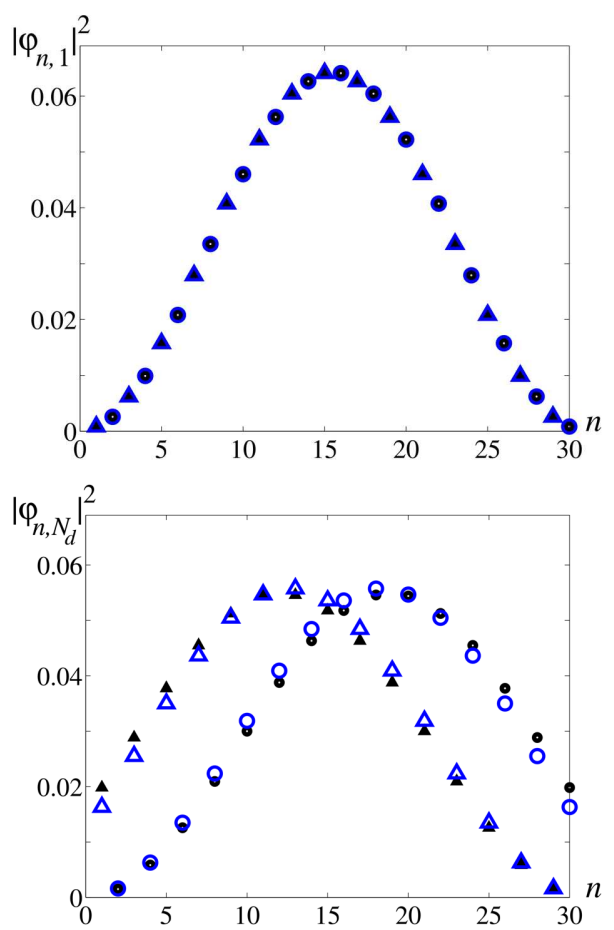

Fig. 3. Dependences of the squared moduli of normalized coefficients of the expansion of the wave functions for a finite polyene chain $\left|\psi_{n, \nu}^{\mathrm{v}}\right|^{2}=\left|\psi_{n, \nu}^{\mathrm{c}}\right|^{2} \equiv\left|\varphi_{n, \nu}\right|^{2}$ on the number of carbon atom $n$ for $\eta=0.133, N_{d}=15$ and $\nu=1$ (upper panel) and $\nu=N_{d}=15$ (lower panel). The black filled symbols are the exact dependences, whereas the blue symbols are the approximate dependences obtained by using relations (11) for $\nu=1$ and (12) for $\nu=15$. The triangles and circles correspond to odd $n=2 m-1$ and even $n=2 m$, respectively

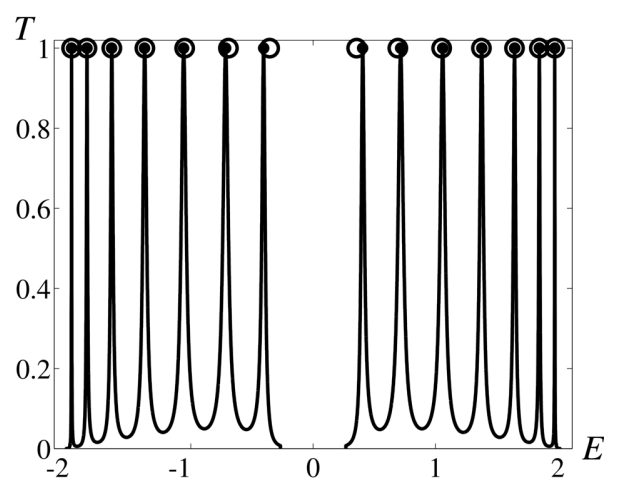

Fig. 4. Dependences $T(E)$ for $A=0.1, N_{d}=7$, and $\eta=$ 0.133. The filled circles correspond to the exact solutions of Eq. (3) and the blank circles correspond to approximation (11)

the HOMO-LUMO gap for the ionized polyene with $\left(2 N_{d}-2\right) \pi$ electrons

$\Delta_{\mathrm{HL}}^{++}=E_{N_{d}}^{\mathrm{v}}-E_{N_{d}-1}^{\mathrm{v}}$ computed with the help of approximation (11) is hardly distinguishable from the exact dependence; see Fig. 2. At the same time, the approximation for $\Delta_{\mathrm{HL}}$ with $x_{N_{d}}$ found with the help of Eqs. (12), (13) practically coincides with the exact dependence; see the red dashed line in Fig. 2.

\subsection{Wave functions}

An example of the application of the obtained approximations for finding the coefficients of expansion of the wave functions for the bounded polyene chain $\left|\psi_{n, \nu}^{\mathrm{v}}\right|^{2}=\left|\psi_{n, \nu}^{\mathrm{c}}\right|^{2} \equiv\left|\varphi_{n, \nu}\right|^{2}$ is shown in Fig. 3. By analyzing the form of these functions presented in Eqs. (5), (6), we can expect that these functions require very accurate approximations for $x_{\nu}$, especially for large $N_{d}$. However, for $\nu=1$, the exact and approximate dependences practically coincide regardless of the use of the simple approximation (11). A more detailed analysis shows that, for $\nu \ll N_{d}$, the dependences of $\psi_{n, \nu}^{\mathrm{v}(\mathrm{c})}$ on $n$ calculated by using approximation (11) are almost indistinguishable from the exact dependences.

As for the function $\left|\varphi_{n, N_{d}}\right|^{2}$, we observe a discrepancy between the exact and approximate values even for $x_{N_{d}}$ found with the help of expression (12), which gives perfect approximations for $\Delta_{\text {HL }}$ presented in the previous subsection. Therefore, to find very accurate dependences of $\psi_{n, \nu}^{\mathrm{v}(\mathrm{c})}$ on $n$ for the roots $x_{\nu}$ with $\nu$ close to $N_{d}$, it might be necessary to find the exact values of the roots numerically by using approximations (10) or (12) as a starting value.

\section{Transmission Spectra for a Bounded Polyene Chain}

A fundamental characteristic of coherent electronic transport in a system formed by a single molecule connecting two metal (or semiconductor) wires is the transmission coefficient $T(E)$, which determines the probability that a stationary incident electron flux passes through the obstacle. The transmission probability is directly related to the current-voltage characteristics $[12,13]$. Within the framework of the Green function formalism, the transmission coefficient $T(E)$ can be expressed $[5,14,15]$ in terms of the Green functions corresponding to the noninteracting left and right wires and the scattering region. In the content of our discussion, the scattering region is the bounded 
polyene chain:

$$
T(E)=\frac{4 \operatorname{Im}\left(A^{l}\right) \operatorname{Im}\left(A^{r}\right)\left|G_{1, N}\right|^{2}}{\left|\left(1-A^{l} G_{1,1}\right)\left(1-A^{r} G_{N, N}\right)-A^{l} A^{r} G_{1, N}^{2}\right|^{2}},
$$

where $A^{l}$ and $A^{r}$ are coupling constants specifying the shift and broadening of molecular levels due to the metal-molecule interaction $[15,16]$, and the matrix elements of the Green function $G_{1,1}, G_{N, N}$, and $G_{1, N}$ are defined in Eq. (8). Since we are mainly interested in the electronic spectrum of polyene, we use a simple model with identical pure imagine coupling constants for the left and right contacts: $A^{l}=A^{r}=-i A$. Thus, for $A \ll 1$, the positions of the peaks of transmission coefficients are specified by the spectrum of the polyene chain placed between the wires.

The results of our calculations of the energy dependences of the transmission coefficient (20) are presented in Fig. 4. It is easy to see that the positions of the exact eigenvalues (marked by the filled circles) are well approximated by the simple relation (11) (blank circles). The maximum deviations from the exact values are attained for the HOMO and LUMO levels. Thus, for these eigenvalues, it is preferable to use approximation (12). The calculated dependences $T(E)$ also clarify the role of the term proportional to $G_{1, N}^{2}$ in the denominator of relation (20). Namely, for all energy values, except the close neighborhoods of eigenvalues $E_{\nu}^{\mathrm{v}(\mathrm{c})}$, this term almost does not affect the behavior of $T(E)$, and the curves presented in Fig. 4 remain identical, if they are calculated with and without the term proportional to $G_{1, N}^{2}$ in the denominator. On the contrary, in the vicinities of $E=E_{\nu}^{\mathrm{v}(\mathrm{c})}$, i.e., in the case of resonant transmission where $\left|G_{1,1}\right|,\left|G_{N, N}\right|,\left|G_{1, N}\right| \gg 1$, its presence becomes crucial.

\section{Conclusions}

In conclusion, we note that, depending on the form of the analyzed characteristics (energy gaps, wave functions, Green functions, etc.), the accurate approximation (12) or the much simpler approximation (11) can be used to find the roots of the characteristic equation $x_{\nu}$. The obtained explicit expressions for the eigenvalues allow us to find approximate analytic expressions for the energy gap and obtain their limits in the cases of large and small numbers of double bonds in finite polyenes. It is also worth noting that the approximations found above can be successfully used as initial values for the exact numerical calculations or as the zero-order approximations for a more detailed analytic analysis of the electrical and transport properties of alternating polyene chains.

\section{APPENDIX.}

\section{HOMO-LUMO gap for particular cases}

It is useful to deduce exact relations for the HOMO-LUMO gap in two simple particular cases: $N_{d}=1$ and $N_{d}=2$. Namely, for $N_{d}=1$, Eq. (3) turns onto $e^{-2 \eta}+2 \cos x=0$, and we immediately get

$E_{1}^{\mathrm{c}}=e^{\eta}, \quad \Delta_{\mathrm{HL}}=2 e^{\eta}$.

For $N_{d}=2$, Eq. (3) can be rewritten in the form $2 e^{-2 \eta} \cos x+$ $4 \cos ^{2} x-1=0$. Thus, we solve the quadratic equation and obtain

$E_{1,2}^{\mathrm{c}}=\mp \frac{e^{-\eta}}{2}+\sqrt{\frac{e^{-2 \eta}}{4}+e^{2 \eta}}, \quad \Delta_{\mathrm{HL}}=e^{-\eta}$.

1. B.S. Hudson. Polyacetylene: Myth and reality. Materials 11, 242 (2018).

2. B.E. Kohler. Electronic properties of linear polyenes. In: Conjugated Polymers, edited by J.L. Bredas, R. Silbey (Kluwer, 1991).

3. B. Kohler, L. Malysheva, A. Onipko. Molecular orbital coefficients and transition dipoles of real polyenes. J. Chem. Phys. 103, 6068 (1995).

4. L. Malysheva, A. Onipko. Exact solution for the Hückel model of heteropolyenes. Synth. Met. 80, 11 (1996).

5. L. Malysheva, A. Onipko. Coherent electron transport in molecular contacts: A case of tractable modeling In: Handbook on Nano- and Molecular Electronics, Chapter 23 (CRC Press, 2007).

6. Y. Tsuji, R. Hoffmann, R. Movassagh, S. Datta. Exponential attenuation of through-bond transmission in a polyene: Theory and potential realizations. J. Chem. Phys. 141, 224311 (2014).

7. T. Kupka, A. Buczek, M. A. Broda, M. Stachów. DFT studies on the structural and vibrational properties of polyenes. J. Mol. Model. 22, 101 (2016).

8. J.E. Lennard-Jones. The electronic structure of some polyenes and aromatic molecules. I-The nature of the links by the method of molecular orbitals. Proc. Roy. Soc. Ser. A 158, 280 (1937).

9. L. Malysheva. Spectral problem for graphene fragments and polyenes: An analytic approach. Phys. Status Solidi B 1600773 (2017).

10. W.P. Su, J.R. Schrieffer, A.J. Heeger. Solitons in polyacetylene. Phys. Rev. Lett. 42, 1698 (1979).

11. Y. Tsuji, E. Estrada. Influence of long-range interactions on quantum interference in molecular conduction. A tight- 
binding (Hückel) approach. J. Chem. Phys. 150, 204123 (2019).

12. R. Landauer. Spatial variation of currents and fields due to localized scatterers in metallic conduction. IBM J. Res. Dev. 1323 (1957).

13. M. Büttiker, Y. Imry, R. Landauer, S. Pinhas. Generalized many-channel conductance formula with application to small rings. Phys. Rev. B 31, 6207 (1985).

14. C. Caroli, R. Combescot, P. Nozières, D. Saint-James. Direct calculation of the tunneling current. J. Phys. C 4916 (1971).

15. S. Datta. Electronic Transport in Mesoscopic Systems (Cambridge Univ. Press, 1995).

16. E.G. Petrov. Modified superexchange model for electron tunneling across the terminated molecular wire. Phys. Status Solidi B 256, 1900092 (2019).

Received 14.09.20

\section{Л.І. Малишева}

АНАЛІТИЧНИЙ АНАЛІЗ

ЕЛЕКТРОННИХ ТА ТРАНСПОРТНИХ

ВЛАСТИВОСТЕЙ ОБМЕЖЕНИХ ПОЛІЕНІВ

На основі наближеного розв'язку характеристичних рівнянь визначено важливі характеристики обмеженого ланцюжка полієну. Отримані наближення та граничні значення хвильових функцій, енергетичної щілини та ін. можна використати для аналізу електронних та транспортних характеристик полієнів, що дозволяє вивчити фундаментальні властивості обмежених альтернованих ланцюжків полієну. Продемонстровано хорошу ефективність запропонованих апроксимацій для чисельного розв'язку характеристичних рівнянь.

$K$ лючов $i$ слова: полієн, функції Гріна, енергетична щілина, коефіцієнт переносу. 